Digital PLL based frequency synthesis:

effect of the loop filter shape on the

required DCO frequency resolution

K. Verheyen, G. Torfs and J. Bauwelinck

This paper is a postprint of a paper submitted to and accepted for publication in Electronics Letters and is subject to Institution of Engineering and Technology Copyright. The copy of record is available at IET Digital Library. [1]

\title{
References
}

1 Verheyen, K., Torfs, G., Bauwelinck, J., 'Digital PLL based frequency synthesis: effect of the loop filter shape on the required DCO frequency resolution', Electron. Lett, 2014, 50, (20), pp. 1425-1427, doi: 10.1049/el.2014.1804 


\section{Digital PLL based frequency synthesis: effect of the loop filter shape on the required DCO frequency resolution}

\section{K. Verheyen, G. Torfs and J. Bauwelinck}

In digital PLLs the finite resolution of digital representation (quantisation) could pose problems, including: jitter peaking and limit-cycle behaviour; both could ruin frequency-stability of a PLL. The resolution of the digitally controlled oscillator (DCO) in the PLL is limited by the smallest dimension available in a given process technology. Currently, one resorts to exhaustive time domain simulations to ensure correct operation of a PLL. Using a behavioural model of a PLL as verification, we investigated how to choose the DCO resolution without resorting to time domain simulations. This letter repeats the conditions on signal statistics for correct behaviour and provides the analysis to estimate the required resolution.

As it turns out, the rms-value of the frequency deviation - neglecting quantisation - is the coarsest possible DCO resolution. One can estimate this value accurately by integration of its power-spectral density, derived from the well-known phase domain model of a PLL.

Introduction: Most modern wireless systems contain a phase locked loop (PLL) for carrier generation, bringing the ability to synthesize a multitude of frequencies from a single reference frequency. During the last decade, digital PLLs became increasingly popular, since the demonstration of a digital PLL that meets the noise specifications of Bluetooth. [1]

The finite resolution of a digitally controlled oscillator (DCO) - part of any digital PLL - can have a huge impact on the performance of the PLL: it can introduce jitter peaking or even result in limit cycle behaviour. Resulting in a decrease of the signal-to-noise ratio and an increase of crosstalk - potentially leading to a failure to comply with a given standard. Without a convenient procedure to determine the DCO frequency resolution, designers must resort to lengthy time domain (behavioural) simulations to ensure no jitter peaking or limit cycle behaviour occurs. Such a situation is far from ideal at an early stage of any design process.

This letter presents an approximation of the required quantisation step. If thermal noise is dominant, this step can be calculated from the in-band noise level, the noise of the oscillator, and the shape of the loop filter. These three specifications allow the estimation of the output jitter - considered to be the main measure of quality for a PLL.

Estimation of the quantisation step - theory: A quantiser is often modelled as a source of additive noise (quantisation noise), despite the fact that there is a causal relation between the quantiser's input and the quantisation noise. Fortunately, under certain conditions this quantisation noise is white, uniform and uncorrelated with the signal to be quantised. So essentially, the quantiser is linearised (Quantisation theorem, QT-II [2, 3]). When such a linearised quantiser is introduced in an otherwise stable loop, no limitcycle behaviour will occur. The loop is not blind for small variations, so it remains closed at all times.

In a $\mathrm{DCO}$, the signal to be quantised is the frequency deviation $\Delta f$ (Fig. 1). Effects of quantisation are ignored to test the satisfaction of QT-II (justification can be found in [2]). Here, $\Delta f$ is considered to be Gaussian, since thermal noise in an electronic system is Gaussian to a good approximation.

For Gaussian signals the conditions for QT-II are approximately satisfied if the standard deviation of these signals is greater than or equal to the quantisation step (assuming a uniform quantiser). This theory agrees with the empirical result presented by Gardner [4]. For the quantisation step that represents the DCO frequency resolution this means:

$$
\underset{\Delta f}{\sigma_{\Delta f}} \geq \underset{(\text { DCO frequency resolution) }}{\Delta f_{\text {res }}}
$$

Estimation of the frequency resolution - phase domain: The analysis in this section starts from the well-known phase domain model of a PLL (Fig. 1), with $G(s)$ the open loop transfer function - also called the loop gain. Two noise sources are sufficient to model the main effects of thermal noise, their power spectral density (PSD) characterises them: (i) $S_{\varphi, d c o}(f)$ represents the thermal noise integrated by the oscillator (free-running) and has the typical $1 / f^{2}$ shape; (ii) $S_{\varphi, \text { ref }}$ accounts for other noise sources and

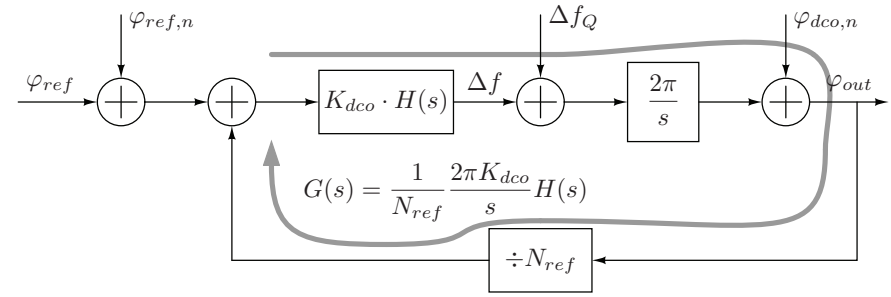

Fig. 1 Conventional phase domain model of a PLL. $K_{\text {dco }}$ is placed before the quantiser, such that the quantisation step corresponds with the DCO frequency resolution $\Delta f_{\text {res. }} \Delta f_{Q}$ : quantisation error; $H(f)$ : loop filter; $G(f): P L L$ open loop transfer function.

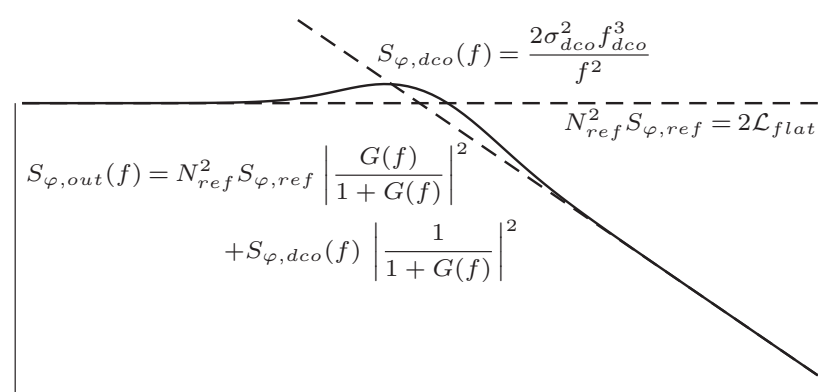

Frequency

Fig. 2 The output phase PSD of the PLL consists of two components - $S_{\varphi \text {,ref }}$ and $S_{\varphi, d c o}(f)$ - tied together by the PLL open loop transfer function $G(f)$.

has a flat spectrum. The PSD of the output phase $S_{\varphi, \text { out }}(f)$ can be written as a linear combination of the PSDs of these two noise sources (Fig. 2).

This model neglects the sampled nature of the PLL, hence it is only valid in case the bandwidth of the loop is small compared to the sampling frequency. To simplify the analysis only thermal noise sources are considered, this means no $1 / f$ noise. Additionally, the integrated phase noise of the reference oscillator is neglected, as it is often made negligible by design.

The standard deviation at the input of the quantiser is estimated by integration of the PSD of the frequency deviation $S_{\Delta f}(f)$ :

$$
\sigma_{\Delta f}^{2}=\int_{0}^{f_{s} / 2} f^{2}\left|\frac{G(f)}{1+G(f)}\right|^{2}\left(N_{r e f}^{2} S_{\varphi, \text { ref }}+S_{\varphi, d c o}(f)\right) d f
$$

To account for the sampled nature of $\Delta f$, the upper limit for integration is $f_{s} / 2$, with $f_{s}$ the sampling frequency. In general $f_{s}$ does not have to be equal to the reference frequency $f_{\text {ref }}$ : it could also be derived from the DCO frequency $f_{d c o}$. Noise sources such as $1 / f$ noise, reference oscillator phase noise and divider noise could be included, through $S_{\varphi, \text { ref }}$ or $S_{\varphi, d c o}(f)$. The effects of digital delay can be included in $G(f)$.

Equation (2) can be used to estimate the required frequency resolution, but does not (yet) provide any design guidance. It will prove to be useful to identify the relation with PLL specifications such as: the output jitter $\sigma_{\varphi, \text { out }}$, the oscillator period jitter $\sigma_{d c o}$ and the in-band phase noise level $\mathcal{L}_{\text {flat }}$. The open loop transfer function $G(f)$ fixes the relation between $\sigma_{\varphi, \text { out }}, \sigma_{d c o}$ and $\mathcal{L}_{\text {flat }}$. The unity-gain frequency $f_{u}$ of $G(f)$ can be chosen such that $\sigma_{\varphi, \text { out }}$ is minimised for a given $\sigma_{d c o}, \mathcal{L}_{\text {flat }}$ and filter shape [5]:

$$
\begin{aligned}
f_{u} & =\sqrt{\frac{\sigma_{\text {dco }}^{2} f_{\text {dco }}^{3}}{\mathcal{L}_{\text {flat }}} \cdot \sqrt{\frac{\beta}{\alpha}}} \\
\sigma_{\varphi, \text { out }}^{2} & =\sqrt{\mathcal{L}_{\text {flat }} \sigma_{\text {dco }}^{2} f_{\text {dco }}^{3}} \sqrt{\alpha \beta}
\end{aligned}
$$

The effect of the filter shape is contained in the factors $\alpha$ and $\beta$, they are defined as:

$$
\alpha=\int_{0}^{+\infty}\left|\frac{G^{(n)}(f)}{1+G^{(n)}(f)}\right|^{2} d f \text { and } \beta=\int_{0}^{+\infty} \frac{1}{f^{2}}\left|\frac{1}{1+G^{(n)}(f)}\right|^{2} d f
$$

where $G^{(n)}(f)=G\left(f / f_{u}\right)$ is called the normalised open loop transfer function, $\left|G^{(n)}(1)\right|=1$. The ratio $f / f_{u}$ is called the normalised frequency. 


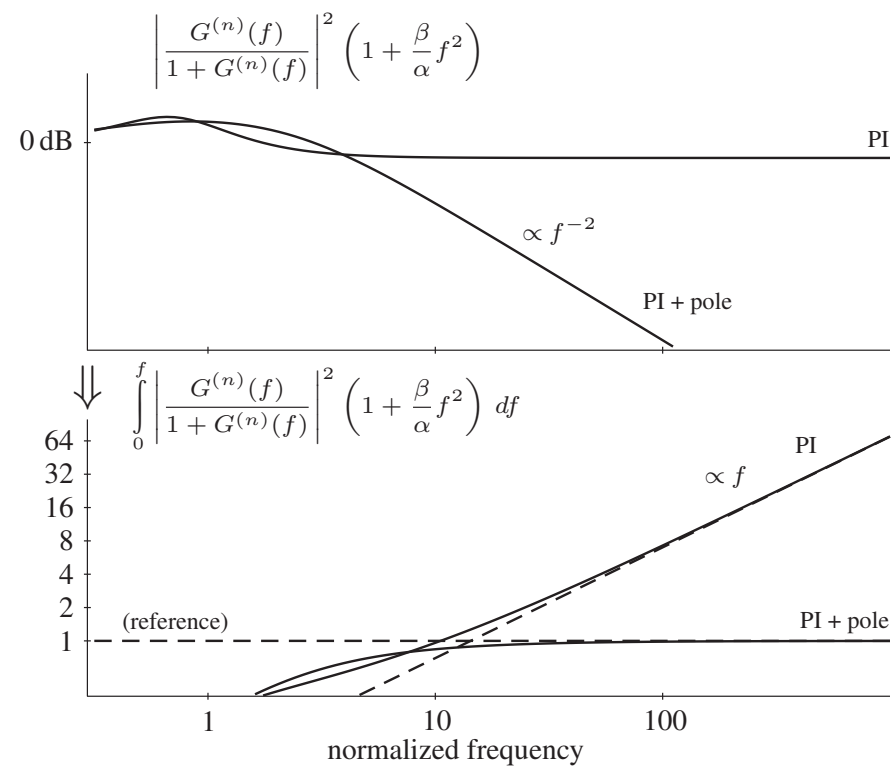

Fig. 3 Comparison of integrand (top) and integral (bottom) from (5) for two types of loop filters. In both filters $P M=60^{\circ}(P I: \rho=\sqrt{3}, \beta / \alpha=0.8 ; P I+$ pole: $\rho=\sqrt{3}+\sqrt{4}, \beta / \alpha=1)$.

Expression (2) can be simplified in the case of an optimal $f_{u}$ :

$$
\sigma_{\Delta f}^{2}=2 \sigma_{d c o}^{2} f_{d c o}^{3} f_{u} \int_{0}^{f_{s} /\left(2 f_{u}\right)}\left|\frac{G^{(n)}(f)}{1+G^{(n)}(f)}\right|^{2}\left(1+\frac{\beta}{\alpha} f^{2}\right) d f
$$

The integrand contains the influence of the loop filter shape on the required DCO frequency resolution. In the next section, this integral is used to show that $\sigma_{\Delta f}^{2}$ is proportional to $f_{s}$ or $f_{u}$.

Case study - two common loop filters: The slope of $G(f)$ at frequencies higher than the unity-gain frequency has a significant impact on the required frequency resolution, especially for PLL's with a narrow bandwidth (high $f_{s} / f_{u}$ ratio). To assess the magnitude of this effect, the integrand (and integral) in equation (5) are compared for the following two normalised open loop transfer functions:

$$
\begin{aligned}
& G^{(n)}(f)=\frac{1}{\sqrt{1+\rho^{2}}} \frac{1+j f \rho}{(j f)^{2}} \quad f \gg 1 \quad \frac{1}{f} \\
& G^{(n)}(f)=\frac{1}{\rho} \frac{1+j f \rho}{(j f)^{2}(1+j f / \rho)} \quad f \gg 1 \quad \frac{1}{f^{2}} \quad \text { PI+pole }
\end{aligned}
$$

with $j$ the imaginary unit and $\rho$ a factor that relates the frequency of other poles and zeroes to the unity-gain frequency. In (6) and (7) the loop filter $H(s)$ (Fig. 1) is a PI-filter and a PI-filter with an additional pole, respectively. In both equations $\rho$ is used to establish a certain phase margin (PM).

The comparison is illustrated in Fig. 3, $\rho$ is chosen such that $\mathrm{PM}=60^{\circ}$ for both filters (common choice). For normalised frequencies greater than 10 , the integrand in (5) remains fairly constant in case of a PI-filter and it decreases proportional to $f^{-2}$ in the case an additional pole is present (Fig. 3-top). Clearly, the integral in (5) will increase proportional to the integration upper limit in case of a PI-filter and it will settle to a certain value in the other case (Fig. 3-bottom). These trends are approximated by:

$$
\begin{aligned}
\sigma_{\Delta f}^{2} \approx 2 \sigma_{d c o}^{2} f_{d c o}^{3} \frac{\rho^{2}}{\sqrt{1+\rho^{2}}+\rho^{2}} \frac{f_{s}}{2} & \propto f_{s} & \text { PI } \\
\sigma_{\Delta f}^{2} \approx 2 \sigma_{d c o}^{2} f_{d c o}^{3} \frac{\rho^{2}+1}{\rho-1} \frac{\pi}{2} f_{u} & \propto f_{u} & \text { PI+pole }
\end{aligned}
$$

they are valid for common practical cases: large $f_{s} / f_{u}$ ratio and a reasonable phase margin.

Clearly, $\sigma_{\Delta f}$ is larger in case of a PI-filter, this means a digital PLL will operate properly with a coarser DCO resolution. Adding more than one additional pole outside the loop bandwidth will provide more suppression of reference noise, but it will not influence $\sigma_{\Delta f}$ much, provided that $f_{s} / f_{u}$ is large.

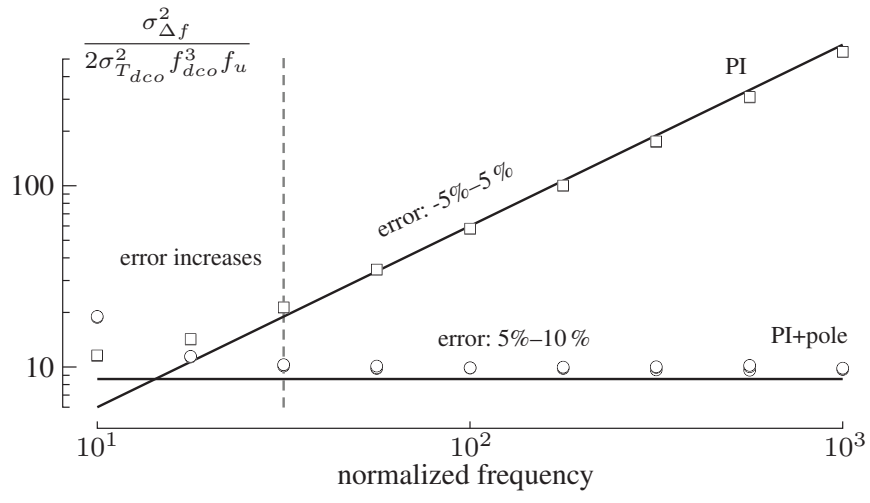

Fig. 4 Estimation of $\sigma_{\Delta f}$ using (8) and (9) agrees with time-domain simulations, for a sufficiently large $f_{s} / f_{u}$ ratio. $\left(f_{\text {dco }} \approx 2.4 \mathrm{GHz} ; f_{\text {ref }}=\right.$ $16 \mathrm{MHz} ; N_{\text {ref }}=150.01 ; N_{s}=16 ; f_{s} \approx f_{d c o} / N_{s}=150 \mathrm{MHz} ; P M=60^{\circ}$; $\left.\sigma_{\varphi, \text { out }}=\left[0.5^{\circ}, 4.7^{\circ}, 45^{\circ}\right]\right)$

Accurate estimate - useful for design guidance: Simulations indicate that the estimate of $\sigma_{\Delta f}$ using (8) and (9) is quite accurate, for a sufficiently high $f_{s} / f_{u}$ ratio ( $>50$ for the filters of the previously section). In the case of a PI filter the error on $\mathrm{f}$ is between $-5 \%$ and $5 \%$, in the other case it is between $5 \%$ and $10 \%$.

In these simulations $f_{\text {ref }}, N_{\text {ref }}$ and $N_{s}$ are kept constant, hence $f_{s}$ has the same value in steady-state. The magnitude of $\mathcal{L}_{\text {flat }}$ and $\sigma_{d c o}$ are adjusted to obtain a certain $f_{s} / f_{u}$ ratio and $\sigma_{\varphi, \text { out }}$.

Conclusion: The proposed equation (2) combined with (3) and (4) link different PLL specifications together. Using this set of equations, the required DCO frequency resolution can be estimated without requiring long time domain simulations. A software package capable of numeric integration is sufficient to make an estimate.

The slope of the loop filter $H(f)$ outside the loop bandwidth has a significant impact on the required DCO frequency resolution. Approximations for two common loop filters (8) and (9) were derived and agreed with time domain simulations of $\sigma_{\Delta f}$. These equations are sufficiently accurate for hand calculations and especially useful for quick estimates.

Equation (2) can be used to include a moderate amount of delay, provided that the PM remains reasonable. Flicker noise could be included as well, but then the lower integration limit must be determined, since integrating down to zero would give rise to an infinite $\sigma_{\Delta f}$ (and $\sigma_{\varphi, \text { out }}$ as well).

K. Verheyen, G. Torfs and J. Bauwelinck (Dept. INTEC, Ghent University -imec-iMinds, Ghent, Belgium)

E-mail: koen.verheyen@intec.ugent.be

\section{References}

1 Staszewski, R., Leipold, D., Muhammad, K., Balsara, P., 'Digitally controlled oscillator (DCO)-based architecture for RF frequency synthesis in a deep-submicrometer CMOS Process', IEEE Trans. Circuits Syst. II, 2003, 50, (11), pp. 815-828, doi: 10.1109/TCSII.2003.819128

2 Widrow, B., 'Statistical analysis of amplitude-quantized sampled-data systems', Transactions of the American Institute of Electrical Engineers, Part II: Applications and Industry, 1961, 79, (6), pp. 555-568, doi: 10.1109/TAI.1961.6371702

3 Widrow, B., Kollár, I., 'Quantization Noise: Roundoff Error in Digital Computation, Signal Processing, Control, and Communications'(Cambridge University Press, Cambridge, UK, 2008).

4 Gardner, F.M., 'Frequency granularity in digital phaselock loops' IEEE Trans. commun., 1996, 44, (6), pp. 749-758, doi: 10.1109/26.506392

5 Gao, X., Klumperink, E., Geraedts P., Nauta, B, 'Jitter Analysis and a Benchmarking Figure-of-Merit for Phase-Locked Loops', IEEE Trans. Circuits Syst. II, Exp. Briefs, 2009, 56, (2), pp. 117-121, 